\title{
Crude Oil Source Identification of Asphalt via ATR-FTIR Approach Combined with Multivariate Statistical Analysis
}

\author{
Ruibo Ren, ${ }^{1}$ Wenmiao Fan, ${ }^{1}$ Pinhui Zhao ${ }^{1},{ }^{1}$ Hao Zhou, ${ }^{1}$ Weikun Meng, ${ }^{1}$ and Ping Ji ${ }^{2}$ \\ ${ }^{1}$ Shandong Provincial Key Laboratory of Road and Traffic Engineering in Colleges and Universities, \\ School of Transportation Engineering, Shandong Jianzhu University, Jinan 250101, China \\ ${ }^{2}$ Shandong Hi-Speed Engineering Testing Co., Ltd., Jinan 250101, China \\ Correspondence should be addressed to Pinhui Zhao; zhaopinhui08@163.com
}

Received 14 February 2020; Revised 15 May 2020; Accepted 3 June 2020; Published 6 July 2020

Guest Editor: Meng Guo

Copyright (c) 2020 Ruibo Ren et al. This is an open access article distributed under the Creative Commons Attribution License, which permits unrestricted use, distribution, and reproduction in any medium, provided the original work is properly cited.

The types of crude oil for producing asphalt have a decisive influence on various performance measures (including aging resistance and durability) of asphalt. To discriminate and predict the crude oil source of different asphalt samples, a discrimination model was established using 12 greatly different infrared (IR) characteristic absorption peaks (CAPs) as predictive variables. The model was established based on diverse fingerprint recognition technologies (such as principal component analysis (PCA) and multivariate logistic regression analysis) by using attenuated total reflectance-Fourier transform infrared spectroscopy (ATRFTIR). In this way, the crude oil source of different asphalt samples can be effectively discriminated. At first, by using PCA, the 12 CAPs in the IR spectra of asphalt samples were subjected to dimension reduction processing to control the variables of key factors. Moreover, the scores of various principal components in asphalt samples were calculated. Afterwards, the scores of principal components were analysed through modelling based on multivariate logistic regression analysis to discriminate and predict the crude oil source of different asphalt samples. The result showed that the logistic regression model shows a favourable goodness of fit, with the prediction accuracy reaching $93.9 \%$ for the crude oil source of asphalt samples. The method exhibits some outstanding advantages (including ease of operation and high accuracy), which is important when controlling the source and quality and improving the performance of asphalt.

\section{Introduction}

Asphalt pavements are widely used: as a black binding material produced from oil, asphalt is widely used as the binder in asphaltic mixtures [1-3]. Due to the differences in origins and production modes of crude oil for producing asphalt, the properties of crude oil exert important influences on the performance of asphalt mixtures, which also lead to significant differences in the performance of the various asphalt produced therewith [4-8].

The conventional performance of the same grade of asphalt is very similar; however, different asphalt exhibit large differences in various aspects, including high- and lowtemperature performance, durability, and fatigue properties, which are considered as external expressions of chemical composition, molecular structure, and transformation of asphalt [9-11]. Furthermore, the study shows that the differences in the composition and structure of asphalt mainly depend on the source of crude oil and refining process of asphalt production. Due to the differences in the geological structure, oil generation conditions, and age, the nature and composition of crude oil in different regions are very different. However, crude oil with similar properties and composition in the same region has similar processing, storage, and transportation options. At the same time, most of the petroleum asphalt is produced by distillation currently, and the molecules in the asphalt retain their original state in the crude oil. Therefore, most of the composition and structure of asphalt are inherited from crude oil; that is to say, the structural performance of asphalt mainly depends on the source of crude oil. Because the asphalt is produced by different types of crude oil, the physical and chemical 
composition information about asphalt is unique. Just like the human fingerprint information, these components which can express the unique structure of asphalt can be called the "oil fingerprint" of asphalt. It is because of the uniqueness of "oil fingerprint" information of asphalt that it is feasible to discriminate the oil fingerprints of asphalt from different crude oil sources [12-16].

At present, as the composition and structure of asphalt are extremely complex, the characterization of its structure requires more high-resolution and high-throughput analysis means and equipment, so there are few reports on the identification and analysis of asphalt oil fingerprints [17]. However, the identification and analysis of marine oil spill fingerprints has always been an issue of widespread concern. Similar to the method and purpose of identifying "oil fingerprints" of oil spills at sea, the purpose of recognising oil fingerprints of asphalt is to attain oil fingerprint information of asphalt through different methods such as physical, chemical, and biological methods [18]. Moreover, by applying multivariate statistical methods (including principal component analysis (PCA) and regression analysis), the chemical composition variables of oil fingerprints are summarised, classified, and discriminated $[19,20]$. On this basis, qualitative and quantitative relationships between data are obtained to distinguish the crude oil source of asphalt, thus effectively controlling their qualities. Meanwhile, some testing methods used in the "oil fingerprint" identification of marine oil spills have been successfully used to analyse the composition and structure of asphalt [21-23]. For example, a gas chromatograph-mass spectrometer (GC-MS) was used to explore the chemical compositions of smoke released by asphalt materials during heating [24, 25]. Gel permeation chromatography (GPC) and thin-layer chromatography (TLC) were used to measure the molecular weights and the composition distributions of asphalt [26-28]. Nuclear magnetic resonance (NMR) and Fourier transform infrared spectroscopy (FTIR) were used to investigate the compositions, structures, and functional groups of asphalt $[29,30]$. In all analytical techniques, compared with other methods (including GC-MS and NMR), which generally show some disadvantages (including high cost, damage to samples, and being laborious and time consuming during analysis), infrared (IR) spectroscopy is the most widely used technique in investigating asphalt materials. The reason is that IR spectroscopy shows many outstanding advantages, including being label-free, rapid, nondestructive, and low-cost, with simple sample preparation [31-33]; however, in the above analysis, the chemical structures of asphalt are qualitatively analysed, mainly aiming at those of a certain or multiple specific asphalt samples while lacking quantitative research into the types of asphalt. The research into discrimination of the types of asphalt, tracing of the production area, and quality control of asphalt has not yet been reported.

Therefore, by utilising attenuated total reflectanceFourier transform infrared spectroscopy (ATR-FTIR), the characteristic functional groups of asphalt from different crude oil source were discriminated and quantitatively analysed. Based on multivariate statistics, PCA and logistic regression analysis were conducted on IR spectral data to establish a discriminant function. An accurate, nondestructive, stable method of discriminating the crude oil source of asphalt samples was explored, which provides a scientific basis for realising reasonable selection, supervision quality, and guaranteed origins of asphalt.

\section{Experimental Raw Materials and Methods}

2.1. Experimental Materials. During the experiment, 33 asphalt samples were purchased from factories in China for producing asphalt. Before being applied, the asphalt samples were sealed in original oxygen-free containers at $5^{\circ} \mathrm{C}$ to prevent the samples from being oxidised. Additionally, all asphalt samples were unprocessed before use. As mentioned in Section 1, the differences in the "oil fingerprint" of asphalt are determined by the crude oil from which it is produced. Due to the same geological structure, oil generation conditions, and age in the same region, the composition and chemical structure of crude oil are also very similar. Therefore, the "oil fingerprints" of asphalt produced by crude oil from the same region are very similar, such as crude oil from the Middle East Gulf region, including Saudi Arabia, Iran, Kuwait, Iraq, and United Arab Emirates, crude oil from South America, including Marry, Poscan, Maya, and Castilla, and crude oil from the Bohai Rim region of China, such as Bohai Bay, Huanxiling, and Caofeidian. The crude oil of 33 asphalt samples came from the above three regions. According to the names of the three regions, the crude oil source of asphalt is divided into three categories: Middle East, South America, and the Bohai Rim region of China. The basic performance measures (penetration ratio (ASTM D5), ductility ratio (ASTM D113), and softening point (ASTM D36)) of asphalt and the crude oil source of asphalt are listed in Table 1. It is worth noting that the last digit of the asphalt number listed in Table 1 represents different sampling batches of the same asphalt.

2.2. FTIR Analysis. Through ATR-FTIR (using a Cary 630 FTIR microscope), the IR spectra of asphalt samples were explored. Within the range of $400-4,000 \mathrm{~cm}^{-1}, 64$ scans were conducted, each at a resolution of $1 \mathrm{~cm}^{-1}$. The samples were placed on the horizontal ATR crystal made of zinc selenide, being subjected to multiple reflections. After each operation, the ATR crystal was cleaned using acetone.

The original spectrum data were first subjected to baseline correction by applying the OMNIC software to eliminate baseline effects. Afterwards, based on the standardised variation diagram of preprocessed spectrum data, the difference in masses of different samples was eliminated.

2.3. Multivariate Statistical Analysis. Through the combination of principal component analysis (PCA) and multiple logistic regression analysis, the infrared spectrum data are analysed to establish the discrimination model of the crude oil source of asphalt. Logistic regression analysis is a multivariate analysis method to analyse and predict attributedependent variables based on single or multiple continuous or attribute-independent variables. Furthermore, each 
TABLE 1: Basic properties and crude oil source of asphalt.

\begin{tabular}{|c|c|c|c|c|c|c|}
\hline $\begin{array}{l}\text { Asphalt } \\
\text { number }\end{array}$ & $\begin{array}{l}\text { Asphalt } \\
\text { name }^{1}\end{array}$ & $\begin{array}{l}\text { Penetration } \\
(0.1 \mathrm{~mm})\end{array}$ & $\begin{array}{c}\text { Ductility }(\mathrm{cm}, \\
\left.10^{\circ} \mathrm{C}\right)\end{array}$ & $\begin{array}{c}\text { Softening point } \\
\left({ }^{\circ} \mathrm{C}\right)\end{array}$ & Source & Category \\
\hline 1 & MM-1 & 79.6 & 65.7 & 47.3 & Middle East (Saudi Arabia) & 1 \\
\hline 2 & Q-1 & 75.4 & $>100$ & 46.5 & South America (Marry \& Poscan) & 2 \\
\hline 3 & SK (BY)-1 & 69.7 & 52.2 & 46.9 & Middle East (Saudi Arabia) & 1 \\
\hline 4 & Q-2 & 61.5 & 39.0 & 48.5 & South America (Marry \& Poscan) & 2 \\
\hline 5 & SL-1 & 63.4 & 47.5 & 47.0 & Middle East (Saudi Arabia) & 1 \\
\hline 6 & CMR-1 & 67.5 & $>100$ & 47.2 & South America (Marry) & 2 \\
\hline 7 & LH-1 & 76.6 & $>100$ & 45.6 & South America (Marry \& Poscan) & 2 \\
\hline 8 & QPK-1 & 61.8 & $>100$ & 46.8 & Middle East (Iran) & 1 \\
\hline 9 & MM-2 & 61.1 & 16.0 & 49.6 & Middle East (Saudi Arabia) & 1 \\
\hline 10 & QP-1 & 68.9 & 33.2 & 48.7 & Middle East (Kuwait) & 1 \\
\hline 11 & JB-1 & 67.3 & 74.0 & 47.5 & South America (Marry \& Poscan) & 2 \\
\hline 12 & SK $(X Y)-1$ & 71.5 & 47.9 & 47.2 & Middle east (Saudi Arabia) & 1 \\
\hline 13 & ZH-1 & 67.4 & 13.5 & 48.1 & South America (Maya \& Castilla) & 2 \\
\hline 14 & JL-1 & 64.2 & 35.6 & 48.2 & Middle East (Kuwait) & 1 \\
\hline 15 & HR-1 & 63.2 & 85.2 & 48.6 & South America (Marry \& Poscan) & 2 \\
\hline 16 & AS-1 & 62.1 & 84.3 & 47.4 & Middle East (Saudi Arabia) & 1 \\
\hline 17 & $\mathrm{XT}-1$ & 62.7 & 23.9 & 48.8 & Middle East (Saudi Arabia) & 1 \\
\hline 18 & $\mathrm{ZH}-2$ & 64.0 & $>100$ & 50.1 & South America (Maya\& Castilla) & 2 \\
\hline 19 & LH-2 & 69.0 & $>100$ & 47.0 & South America (Marry \& Poscan) & 2 \\
\hline 20 & QL-1 & 64.0 & 21.3 & 50.0 & Middle east (Kuwait) & 1 \\
\hline 21 & $\mathrm{KL}-1$ & 79.8 & $>100$ & 47.2 & $\begin{array}{c}\text { Bohai Rim region of China (Bohai } \\
\text { Bay) }\end{array}$ & 3 \\
\hline 22 & KL-2 & 61.0 & 57.8 & 49.5 & $\begin{array}{c}\text { Bohai Rim region of China (Bohai } \\
\text { Bay) }\end{array}$ & 3 \\
\hline 23 & SL-2 & 65.0 & 30.0 & 49.2 & Middle East (Saudi Arabia) & 1 \\
\hline 24 & SK (NB)-1 & 71.0 & 47.9 & 48.4 & Middle East (Saudi Arabia) & 1 \\
\hline 25 & Q-3 & 75.0 & $>100$ & 47.3 & South America (Marry \& Poscan) & 2 \\
\hline 26 & DSZ-1 & 69.0 & $>100$ & 50.0 & $\begin{array}{c}\text { Bohai Rim region of China } \\
\text { (Huanxiling) }\end{array}$ & 3 \\
\hline 27 & CMR-2 & 68.2 & $>100$ & 47.9 & South America (Marry) & 2 \\
\hline 28 & JB-2 & 64.6 & 74.0 & 46.3 & South America (Marry \& Poscan) & 2 \\
\hline 29 & $\mathrm{ZH}-3$ & 63.5 & $>100$ & 51.2 & South America (Maya \& Castilla) & 2 \\
\hline 30 & LH-3 & 68.7 & $>100$ & 47.9 & South America (Marry \& Poscan) & 2 \\
\hline 31 & DSZ-2 & 78.4 & $>100$ & 48.6 & $\begin{array}{c}\text { Bohai Rim region of China } \\
\text { (Huanxiling) }\end{array}$ & 3 \\
\hline 32 & SZ-1 & 61.9 & 57.8 & 49.3 & $\begin{array}{c}\text { Bohai Rim region of China (Bohai } \\
\text { Bay) }\end{array}$ & 3 \\
\hline 33 & SZ-2 & 68.0 & $>100$ & 50.9 & $\begin{array}{c}\text { Bohai Rim region of China (Bohai } \\
\text { Bay) }\end{array}$ & 3 \\
\hline
\end{tabular}

${ }^{1}$ In this column, 1,2 , and 3 indicate the different sampling batches of the same asphalt.

variable is required to be independent of each other in variable screening and parameter estimation. In many studies, there is a certain degree of linear dependence between their variables, which is called multicollinearity. This multiple collinear relationship may increase the mean square error and standard error of the estimated parameters, which leads to the instability of the analysis results of the logistic regression model. The main reason for the problem of multicollinearity is the overlap of information. However, PCA can reduce the repeatability of information and achieve the purpose of eliminating multicollinearity by extracting independent principal components from explanatory variables.

For this reason, this study used a multinomial logistic regression model based on PCA to improve the discrimination accuracy of the model. First of all, the PCA was used to reduce the dimension of the CAPs variables of the infrared spectrum, so that the variables with strong correlation were integrated into the same principal components. The principal components were independent of each other; thus, the multiple collinear relationship between variables was eliminated. Then, by using these principal components as independent variables, the discriminant model of crude oil source of asphalt was obtained by logistic regression analysis.

2.3.1. PCA Analysis. PCA refers to a simplification of multidimensional data to several relevant variables (principal components) through a dimension reduction approach. Each principal component reflects most of the information of original variables, and the contained information is not repeated. PCA can compress countless information and simplify complex problems [34]. The modelling process of PCA is as follows: 
(1) Calculation of the correlation coefficient matrix:

$$
R=\left[\begin{array}{cccc}
r_{11} & r_{12} & \cdots & r_{1 p} \\
r_{21} & r_{22} & \cdots & r_{2 p} \\
\vdots & \vdots & & \vdots \\
r_{p 1} & r_{p 2} & \cdots & r_{p p}
\end{array}\right],
$$

where $r_{i j}(i, j=1,2, \ldots, p)$ refers to the correlation coefficient of original variables $X_{i}$ and $X_{j}, r_{i j}=r_{j i}$, which can be calculated by using the following formula:

$$
r_{i j}=\frac{\sum_{k=1}^{n}\left(X_{k i}-\bar{X}_{i}\right)\left(X_{k j}-\bar{X}_{j}\right)}{\sqrt{\sum_{k=1}^{n}\left(X_{k i}-\bar{X}_{i}\right)^{2} \sum_{k=1}^{n}\left(X_{k j}-\bar{X}_{j}\right)^{2}}} .
$$

(2) Calculating eigenvalues and eigenvectors:

The characteristic equation $|\lambda I-R|=0$ was solved. Generally, the eigenvalues were calculated by using the Jacobi method and, in descending order are $\lambda_{1} \geq \lambda_{2} \geq \cdots \geq \lambda_{p} \geq 0$. The eigenvectors $e_{i}(i=1,2$, $\ldots, p)$ corresponding to eigenvalue $\lambda_{1}$ were separately calculated, satisfying $\left\|e_{i}\right\|=1$, that is, $\sum_{j=1}^{p} e_{\mathrm{ij}}^{2}=1$, where $e_{i j}$ denotes the $j^{\text {th }}$ component of vector $e_{i}$.

(3) Calculating contribution and cumulative contribution of principal components:

$$
\text { contribution : } \frac{\lambda_{i}}{\sum_{k=1}^{p} \lambda_{k}}, \quad(i=1,2, \ldots, p),
$$

cumulative contribution : $\frac{\sum_{\mathrm{k}=1}^{i} \lambda_{k}}{\sum_{k=1}^{p} \lambda_{k}}, \quad(i=1,2, \ldots, p)$.

In general, the eigenvalues with the cumulative contribution not lower than $70 \%$ are taken. $\lambda_{1}, \lambda_{2}, \ldots, \lambda_{m}$ are the corresponding first, second, ..., $m^{\text {th }}(m \leq p)$ principal components.

(4) Calculating the loads of principal components:

$$
l_{i j}=p\left(Z_{i}, X_{j}\right)=\sqrt{\lambda_{i} e_{i j}} . \quad(i=1,2, \ldots, p) .
$$

(5) Scores of various principal components:

$$
Z=\left[\begin{array}{cccc}
z_{11} & z_{12} & \cdots & z_{1 m} \\
z_{21} & z_{22} & \cdots & z_{2 m} \\
\vdots & \vdots & & \vdots \\
z_{n 1} & z_{n 2} & \cdots & z_{n m}
\end{array}\right] .
$$

2.3.2. Logistic Regression Analysis. Logistic regression is a multivariate analysis method for investigating the relationship between binominal or multinomial observation results (dependent variable) and influencing factors (independent variable), belonging to probabilistic nonlinear regression methods. The logistic regression when the dependent variable only shows two or more states belongs to binomial logistic regression and multinomial logistic regression, respectively $[35,36]$. For discriminating and classifying the crude oil of asphalt, multinomial logistic regression is applied to conduct data analysis, owing to the crude oil of asphalt being sourced from the Bohai Rim region of China, South America, and the Middle East.

\section{(1) Model fitting:}

For multinomial logistic regression, a certain level of dependent variables is defined as the reference level herein. Compared with the other levels, $i-1$ ( $i$ refers to the number of dependent variables) generalised logistic regression models were fitted. By taking three-level dependent variables as an example, it is supposed that the values of dependent variables are 1 , 2, and 3: the probabilities corresponding to the values are $\pi_{1}, \pi_{2}$, and $\pi_{3}$, respectively. Based on $m$-independent variables, two models are fitted as follows:

$$
\begin{aligned}
& \operatorname{logit} \frac{\pi_{1}}{\pi_{3}}=\alpha_{1}+\beta_{11} X_{1}+\beta_{12} X_{2}+\ldots+\beta_{1 m} X_{m}, \\
& \operatorname{logit} \frac{\pi_{2}}{\pi_{3}}=\alpha_{2}+\beta_{21} X_{1}+\beta_{22} X_{2}+\ldots+\beta_{2 m} X_{m} .
\end{aligned}
$$

(2) Meaning of regression parameters:

For multinomial logistic regression, each independent variable contains $(m-1)$ parameters. The parameter $\beta_{1 m}$ represents an independent variable $x_{m}$ that changes one unit on the premise that other independent variables remain unchanged, and it reflects the variation of the log-odds ratio (OR) of class $i$. The OR is subjected to logarithmic transformation to obtain the linear mode $\left(\ln \left(p_{i} / 1-p_{i}\right)=\right.$ $\left.\beta_{0}+\beta_{1} X_{1}+\beta_{2} X_{2}+\ldots+\beta_{n} X_{n}\right)$ of the logistic regression model.

\section{Results and Discussion}

3.1. Establishment of Discrimination Indices for Crude Oil Source of Asphalt. FTIR is an important means of identifying organic compounds. When irradiating organics using the IR light, the molecules absorb the IR light leading to vibrational energy level transition, and different chemical bonds or functional groups show diverse absorption frequencies. The contents of various materials are reflected in their IR absorption spectra, which can be quantitatively analysed according to peak location and absorption intensity. The structural composition of asphalt is complex, and asphalt shows significant differences in behaviour. For these reasons, it fails to effectively characterize the difference of behaviours of asphalt from different crude oil only by quantitatively comparing the peak areas of IR spectrograms. Therefore, by observing the shapes and locations of IR spectrograms, 12 significant characteristic absorption peaks (CAPs) were selected to analyse the transmittances of absorption peaks. 
The IR absorption spectra of 33 asphalt samples are similar. By using the mean value method, the mutual mode of the IR spectrogram of all asphalt samples was constructed (Figure 1): the assignments of 12 characteristics peaks are as follows: the strong absorption peaks around $2850 \mathrm{~cm}^{-1}$ and $2920 \mathrm{~cm}^{-1}$ are triggered by the stretching vibration of $\mathrm{CH}_{2}$, and a very weak absorption peak around $1700 \mathrm{~cm}^{-1}$ is induced by the stretching vibration of $\mathrm{C}=\mathrm{O}$. Moreover, the vibration of the benzene ring leads to the absorption peak in the vicinity of $1600 \mathrm{~cm}^{-1}$, and the absorption peaks at $1380 \mathrm{~cm}^{-1}$ and $1460 \mathrm{~cm}^{-1}$ are caused by the bending vibration of $\mathrm{CH}_{3}$. The fingerprint region appears below $1300 \mathrm{~cm}^{-1}$, in which the absorption peaks at $1166 \mathrm{~cm}^{-1}$ and $1032 \mathrm{~cm}^{-1}$ are triggered by the stretching vibrations of $\mathrm{C}=\mathrm{S}$ and $\mathrm{S}=\mathrm{O}$, respectively. The stretching vibration of $\mathrm{CH}$ results in a weak absorption peak around $969 \mathrm{~cm}^{-1}$, while the absorption peaks at $872 \mathrm{~cm}^{-1}$ and $812 \mathrm{~cm}^{-1}$ are induced by vibrations of an isolated hydrogen and two adjacent hydrogen atoms on the benzene ring, respectively. Additionally, the absorption peak at $723 \mathrm{~cm}^{-1}$ is also caused by the stretching vibration of $\mathrm{CH}_{2}$.

3.2. Analysis of Predictive Variables Based on Descriptive Statistics. The IR spectra of all asphalt samples are similar, and it is difficult to distinguish the differences among asphalt samples by comparing spectrograms alone. Hence, 12 significantly different CAPs were selected from the spectrograms to describe the transmittances of absorption peaks based on descriptive statistics. From two aspects of centralised location (including indices such as average and median) and degree of dispersion (including indices such as extreme value), the samples are described so as to reflect spectrographic data (Table 2).

In Table 2, according to the analysis result of descriptive statistics on the transmittances of 12 CAPs, it can be seen that the asphalt produced by crude oil from the Bohai Rim region of China showed a larger transmittance. By contrast, the transmittances of asphalt produced by crude oil from the Middle East and South America were consistently low. However, it is impossible to distinguish the oil source of asphalt based on the descriptive statistics of infrared spectral transmittance of asphalt. Therefore, it is necessary to introduce multivariate statistical analysis methods, such as multinomial logistic regression analysis based on PCA described in Section 2.3.

3.3. Correlation Analysis of Predictive Variables. Correlation analysis aims to explore the correlation among multiple variables, which is also an important parameter for evaluating the fingerprint variables of asphalt [37]. In order to further evaluate whether the selected 12 variables were of sufficient significance to the prediction model, a correlation analysis of the 12 CAP variables was required. Generally, correlation analysis is conducted by applying Pearson and Spearman correlation coefficients. The Pearson correlation

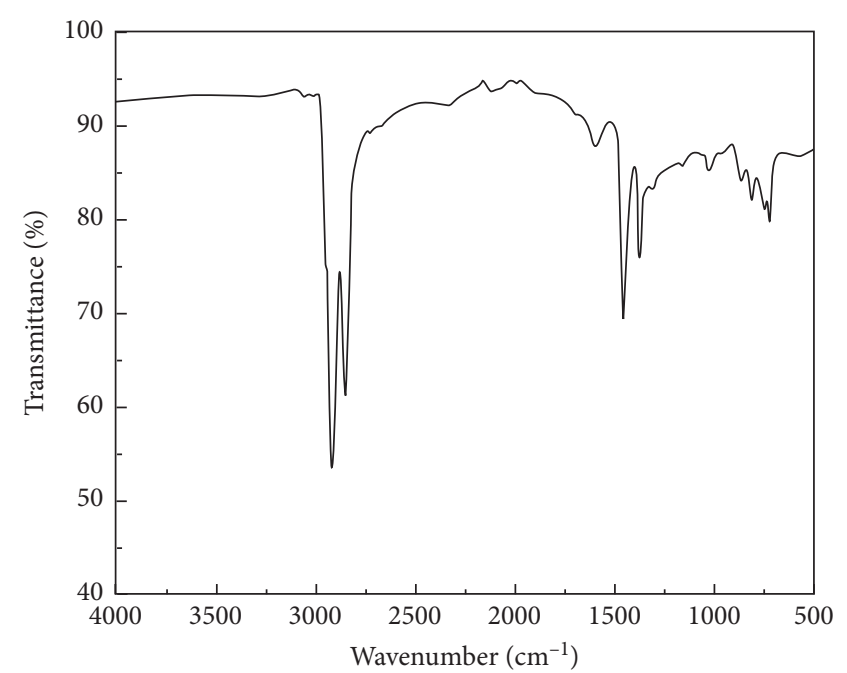

Figure 1: The common mode of infrared fingerprints of the asphalt.

coefficient is generally applicable to data satisfying a normal distribution, and the Spearman correlation coefficient is employed for data that do not satisfy a normal distribution. Therefore, before the correlation test, it is necessary to test the normal distribution of 12 variables to determine the appropriate correlation test method.

By using the skewness-kurtosis test method, whether the transmittances of the 12 CAPs of 33 asphalt samples conform to a normal distribution was assessed, and through the K-S test as an auxiliary analysis method, the accuracy of the test results was ensured [38, 39]. The 12 variables were processed by importing them into SPSS19 (Tables 3 and 4).

It can be seen from Table 3 that the values of skewness and kurtosis of transmittances of the 12 CAPs of all asphalt samples produced by three origins of oil fluctuate within a certain small positive and negative range around zero. It can be further seen from Table 4 that the asymptotic significances of the 12 variables all exceed 0.05 . Moreover, based on the result of the skewness-kurtosis test, it can be considered that the 12 variables of 33 asphalt samples all conform to a normal distribution, which provides a basis for determining the method for testing correlation among variables. Therefore, the Pearson correlation coefficient is used to analyse the correlation between variables (Table 5).

As shown in Table 5, the IR CAP at $2850 \mathrm{~cm}^{-1}$ showed a significant correlation with those at 2920, 1460, and $723 \mathrm{~cm}^{-1}$, respectively. Additionally, there are significant correlations between each IR CAP at 1700, 1600, 1460, 1380, $1166,1032,969,872,812$, and $723 \mathrm{~cm}^{-1}$. Moreover, multiple CAPs exhibited a high correlation. The aforementioned CAPs with high correlation covered all 12 CAPs. This showed that the 12 selected CAPs contained most of the fingerprint information about the asphalt, thus providing a basis for selecting variables capable of discriminating the different crude oil sources of asphalt. 
TABle 2: Descriptive statistics analysis of the 12 CAPs.

\begin{tabular}{|c|c|c|c|c|c|}
\hline Statistical indicators & Oil source & Average value & Median value & Maximum value & Minimum value \\
\hline \multirow{3}{*}{$2920^{-1}$} & Middle East & 52.10 & 51.62 & 55.46 & 49.83 \\
\hline & South America & 55.85 & 54.20 & 61.02 & 53.29 \\
\hline & Bohai Rim region of China & 54.39 & 53.29 & 56.64 & 53.23 \\
\hline \multirow{3}{*}{$2850^{-1}$} & Middle East & 59.82 & 59.51 & 63.60 & 58.43 \\
\hline & South America & 63.26 & 62.39 & 67.38 & 60.74 \\
\hline & Bohai Rim region of China & 62.97 & 62.36 & 64.88 & 61.69 \\
\hline \multirow{3}{*}{$1700^{-1}$} & Middle East & 91.07 & 91.14 & 91.96 & 89.28 \\
\hline & South America & 91.46 & 91.50 & 92.29 & 90.83 \\
\hline & Bohai Rim region of China & 92.92 & 92.41 & 94.06 & 92.29 \\
\hline \multirow{3}{*}{$1600^{-1}$} & Middle East & 87.31 & 87.37 & 88.25 & 85.10 \\
\hline & South America & 87.67 & 87.49 & 88.74 & 87.20 \\
\hline & Bohai Rim region of China & 90.66 & 90.26 & 91.63 & 90.08 \\
\hline \multirow{3}{*}{$1460^{-1}$} & Middle East & 68.71 & 68.64 & 70.10 & 67.64 \\
\hline & South America & 69.81 & 69.81 & 71.68 & 68.27 \\
\hline & Bohai Rim region of China & 72.03 & 72.18 & 72.24 & 71.67 \\
\hline \multirow{3}{*}{$1380^{-1}$} & Middle East & 75.51 & 75.48 & 76.95 & 74.08 \\
\hline & South America & 76.11 & 76.12 & 77.26 & 74.77 \\
\hline & Bohai Rim region of China & 78.51 & 78.50 & 78.96 & 78.07 \\
\hline \multirow{3}{*}{$1166^{-1}$} & Middle East & 85.26 & 85.41 & 86.33 & 83.43 \\
\hline & South America & 85.88 & 85.91 & 86.84 & 84.65 \\
\hline & Bohai Rim region of China & 89.48 & 89.10 & 90.25 & 89.08 \\
\hline \multirow{3}{*}{$1032^{-1}$} & Middle East & 84.37 & 84.49 & 86.35 & 82.29 \\
\hline & South America & 85.78 & 86.30 & 86.80 & 82.57 \\
\hline & Bohai Rim region of China & 90.10 & 89.81 & 90.68 & 89.81 \\
\hline \multirow{3}{*}{$969^{-1}$} & Middle East & 86.53 & 86.72 & 87.73 & 84.97 \\
\hline & South America & 87.28 & 87.22 & 88.10 & 85.98 \\
\hline & Bohai Rim region of China & 90.33 & 90.15 & 90.69 & 90.14 \\
\hline \multirow{3}{*}{$872^{-1}$} & Middle East & 83.74 & 83.93 & 84.64 & 81.00 \\
\hline & South America & 84.86 & 84.67 & 86.09 & 83.79 \\
\hline & Bohai Rim region of China & 88.97 & 88.53 & 89.86 & 88.50 \\
\hline \multirow{3}{*}{$812^{-1}$} & Middle East & 80.73 & 81.12 & 81.85 & 77.23 \\
\hline & South America & 82.70 & 82.79 & 83.88 & 80.40 \\
\hline & Bohai Rim region of China & 88.63 & 88.24 & 89.43 & 88.21 \\
\hline \multirow{3}{*}{$723^{-1}$} & Middle East & 78.18 & 78.14 & 80.74 & 76.17 \\
\hline & South America & 81.32 & 81.38 & 82.70 & 78.25 \\
\hline & Bohai Rim region of China & 85.61 & 84.90 & 87.15 & 84.78 \\
\hline
\end{tabular}

TABLe 3: The skewness-kurtosis test of the 12 CAPs.

\begin{tabular}{lcc}
\hline Statistical indicators $\left(\mathrm{cm}^{-1}\right)$ & Skewness & Kurtosis \\
\hline 2920 & 1.123 & 1.279 \\
2850 & 0.797 & 0.130 \\
1700 & 0.713 & 1.241 \\
1600 & 1.120 & 1.379 \\
1460 & 0.509 & -1.149 \\
1380 & 0.509 & -0.728 \\
1166 & 1.129 & 0.600 \\
1032 & 0.737 & -0.213 \\
969 & 0.926 & 0.074 \\
872 & 1.052 & 0.735 \\
812 & 0.975 & 0.245 \\
723 & 0.651 & 0.459 \\
\hline
\end{tabular}

\subsection{Establishment of Logistic Regression and Discriminant Model Based on PCA}

3.4.1. PCA on All Variables. According to the correlation analysis of variables, it can be seen that the information contained in the 12 CAPs shows a certain repeatability. PCA not only can remove repeated information but can retain key information, thus realising dimension reduction. Furthermore, it makes the modelling for logistic regression and discrimination more reliable due to reducing the disturbance caused by accidental factors.

The transmittances of the 12 CAPs of 33 asphalt samples are input into the SPSS19 software for PCA. The results are displayed in Table 6 and Figure 2. As shown in Table 6, there 


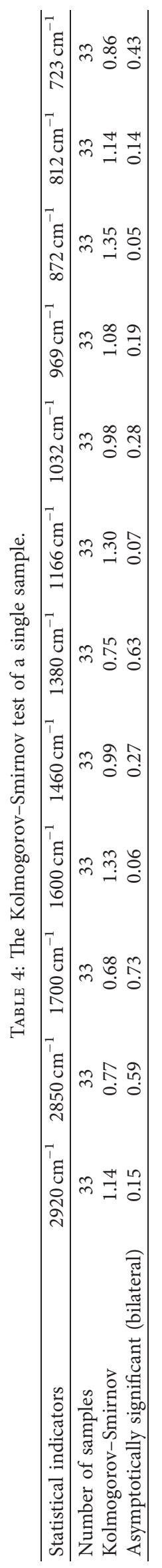




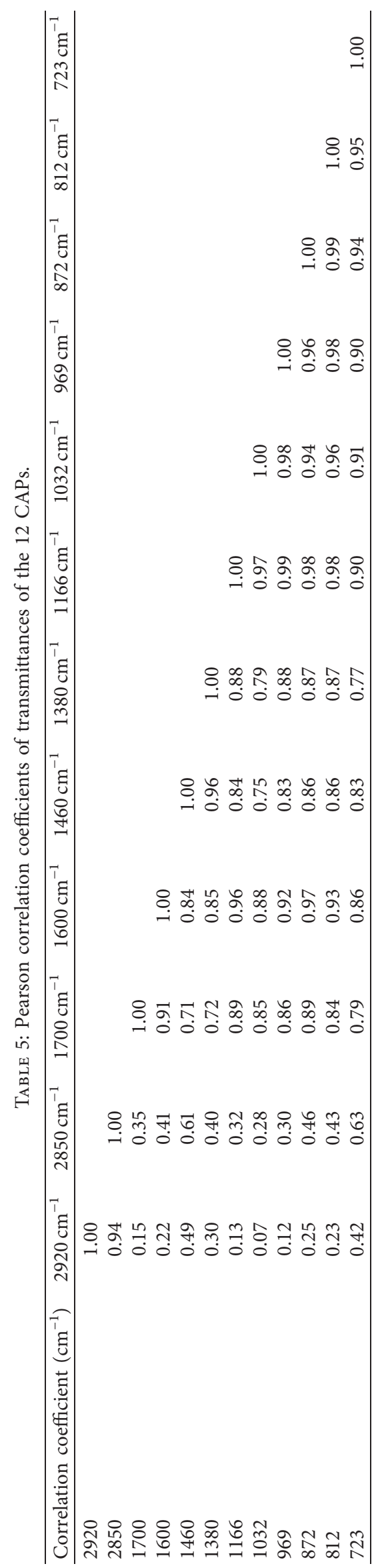


TABLE 6: Result of principal component analysis.

\begin{tabular}{lccc}
\hline Number of principal components & Eigenvalue & Variance contribution (\%) & Cumulative variance contribution (\%) \\
\hline 1 & 9.319 & 77.658 & 77.658 \\
2 & 1.860 & 15.498 & 93.156 \\
3 & 1.017 & 3.508 & 96.664 \\
4 & 0.255 & 2.123 & 98.787 \\
5 & 0.081 & 0.677 & 99.464 \\
6 & 0.031 & 0.256 & 99.720 \\
7 & 0.019 & 0.159 & 99.879 \\
8 & 0.006 & 0.051 & 99.931 \\
9 & 0.004 & 0.033 & 99.964 \\
10 & 0.003 & 0.021 & 99.985 \\
11 & 0.002 & 0.013 & 99.998 \\
12 & 0.000 & 0.002 & 100.000 \\
\hline
\end{tabular}

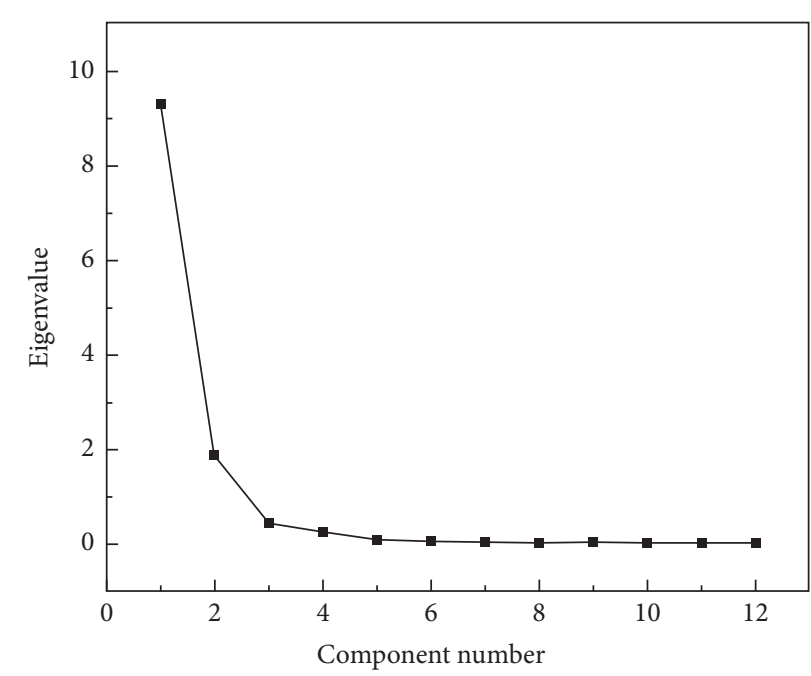

FIgURe 2: Analysis of the scree plot of principal components.

are three principal components whose eigenvalues exceed one. The first, second, and third principal components explain $77.658 \%, 15.498 \%$, and $3.508 \%$ of the nature of the original variables, respectively. The cumulative variance contribution of the three principal components is $96.664 \%$ (research shows that there is a high explanation rate when the cumulative contribution is higher than $70 \%)$. It can be seen from the scree plot (Figure 2) that the broken lines of the first three principal components are steep while later tending to become shallower. This further indicates that it is appropriate to extract the three principal components (PCA1, PCA2, and PCA3). According to the correlation coefficients between the principal component and the original variables, the principal components $Y_{1}, Y_{2}$, and $Y_{3}$ are separately expressed as follows:

$$
\begin{aligned}
Y_{1}= & 0.035 x_{1}+0.055 x_{2}+0.095 x_{3}+0.103 x_{4}+0.098 x_{5}+0.097 x_{6}+0.104 x_{7}+0.100 x_{8}+0.104 x_{9} \\
& \quad+0.106 x_{10}+0.106 x_{11}+0.102 x_{12}, \\
Y_{2}= & 0.503 x_{1}+0.449 x_{2}-0.088 x_{3}-0.056 x_{4}+0.128 x_{5}-0.001 x_{6}-0.115 x_{7}-0.138 x_{8}-0.120 x_{9} \\
& -0.042 x_{10}-0.049 x_{11}+0.069 x_{12}, \\
Y_{3}= & -0.025 x_{1}+0.393 x_{2}+0.527 x_{3}+0.049 x_{4}-0.781 x_{5}-0.997 x_{6}+0.004 x_{7}+0.305 x_{8}-0.025 x_{9} \\
& +0.129 x_{10}+0.069 x_{11}+0.471 x_{12},
\end{aligned}
$$

where $x_{1}, x_{2}, \ldots, x_{12}$ represent the transmittances of CAPs at $2920,2850,1700,1600,1460,1380,1166,1032,969,872$, 812 , and $723 \mathrm{~cm}^{-1}$, respectively.
3.4.2. The Process and Result of Multinomial Logistic Analysis. By substituting the transmittances of the 12 CAPs of 33 asphalt samples into formulae (9-11), the scores of the 
TABLE 7: The scores of each principal component.

\begin{tabular}{|c|c|c|c|c|}
\hline Asphalt number & Asphalt name ${ }^{2}$ & PCA1 & PCA2 & PCA3 \\
\hline 1 & MM-1 & -0.446 & -0.840 & -0.024 \\
\hline 2 & Q-1 & -0.537 & 0.876 & 1.484 \\
\hline 3 & SK (BY)-1 & -0.870 & -0.225 & -0.392 \\
\hline 4 & Q-2 & -0.383 & 0.118 & -1.233 \\
\hline 5 & SL-1 & -0.986 & 0.240 & -0.471 \\
\hline 6 & CMR-1 & -1.796 & 0.963 & -0.386 \\
\hline 7 & LH-1 & -0.865 & -1.151 & 0.438 \\
\hline 8 & QPK-1 & -0.123 & -0.995 & -0.555 \\
\hline 9 & MM-2 & -1.042 & -0.455 & -0.089 \\
\hline 10 & QP-1 & -0.593 & -1.212 & -0.046 \\
\hline 11 & JB-1 & -0.742 & -1.037 & -0.711 \\
\hline 12 & SK (XY)-1 & -1.152 & -0.335 & 0.338 \\
\hline 13 & $\mathrm{ZH}-1$ & -0.470 & -0.919 & -0.641 \\
\hline 14 & JL-1 & -0.113 & -0.717 & -1.306 \\
\hline 15 & HR-1 & -0.523 & -0.757 & 0.423 \\
\hline 16 & AS-1 & -0.316 & -0.098 & 1.739 \\
\hline 17 & XT-1 & -0.268 & 0.156 & 2.187 \\
\hline 18 & $\mathrm{ZH}-2$ & 0.418 & 2.666 & -0.827 \\
\hline 19 & LH-2 & -0.434 & 2.149 & -1.062 \\
\hline 20 & QL-1 & -0.451 & 0.336 & 0.688 \\
\hline 21 & KL-1 & 0.175 & -0.225 & -0.545 \\
\hline 22 & KL-2 & -0.153 & 0.221 & 0.312 \\
\hline 23 & SL-2 & 0.627 & 1.081 & 0.147 \\
\hline 24 & SK (NB)-1 & -0.268 & 0.156 & 2.187 \\
\hline 25 & Q-3 & 0.418 & 2.666 & -0.827 \\
\hline 26 & DSZ-1 & 0.175 & -0.225 & -0.545 \\
\hline 27 & CMR-2 & -0.153 & 0.221 & 0.312 \\
\hline 28 & JB-2 & 1.536 & -0.789 & -0.679 \\
\hline 29 & $\mathrm{ZH}-3$ & 1.626 & -0.611 & -1.122 \\
\hline 30 & LH-3 & 2.273 & 0.072 & 1.503 \\
\hline 31 & DSZ-2 & 1.536 & -0.789 & -0.679 \\
\hline 32 & SZ-1 & 1.626 & -0.611 & -1.122 \\
\hline 33 & SZ-2 & 2.273 & 0.072 & 1.503 \\
\hline
\end{tabular}

${ }^{2}$ In this column, 1,2 , and 3 indicate the different sampling batches of the same asphalt.

three principal components can be calculated (Table 7). Moreover, the scores of the principal components are taken as factors, and three kinds of origins of asphalt are considered as dependent variables. Among them, the crude oil from the Bohai Rim region of China is regarded as a reference group to establish a multinomial logistic regression model based on principal components. On this basis, the parameters of the three principal components used for the logistic regression model are obtained.

Based on the parameter from regression, the logistic regression model can be obtained as follows:

$$
\begin{aligned}
& \operatorname{logit} p_{1}=17.130-40.988 Y_{1}+5.758 Y_{2}-1.788 Y_{3}, \\
& \operatorname{logit} p_{2}=22.543-21.163 Y_{1}+9.509 Y_{2}+0.024 Y_{3}, \\
& \operatorname{logit} p_{3}=0 \text { (reference group), }
\end{aligned}
$$

where $p_{1}, p_{2}$, and $p_{3}$ refer to the probabilities of crude oil sources (the Middle East, South America, and Bohai Rim region of China) of asphalt and $Y_{1}, Y_{2}$, and $Y_{3}$ denote the first, second, and third principal components, respectively.

By substituting expressions (9), (10), and (11) into expression (12), the expression (formula (13)) for characterising the relationship between the logistic regression model and the 12 variables can be acquired. During discrimination and prediction, the probabilities of crude oil sources of asphalt can be separately acquired by substituting the transmittances of the 12 CAPs of the asphalt. The maximum probability corresponds to the predicted origin: 
TABLE 8: The test of the logistic regression model.

\begin{tabular}{lccc}
\hline \multirow{2}{*}{ Test of goodness of fit } & Coefficient & Chi-square & Significance level \\
& Pearson & 5.502 & 1 \\
& Deviance & 6.078 & 1 \\
\hline \multirow{2}{*}{ Test of pseudo $R$-squared } & Cox and Snell & Nagelkerke & McFadden \\
& 0.849 & Chi-square & 0.971 \\
\hline & Parameter & 13.521 & Significance level \\
Test of likelihood ratio & B & 47.335 & 0.001 \\
& F1 & 8.554 & 0.000 \\
& F2 & 3.389 & 0.014 \\
\hline
\end{tabular}

TABLE 9: The discrimination result of multinomial logistic regression.

\begin{tabular}{lcccc}
\hline Observed value & & \multicolumn{2}{c}{ Predicted value } \\
Middle East & South America & Bohai Rim region of China & Discrimination accuracy (\%) \\
\hline Middle East & 14 & 1 & 0 & 93.3 \\
South America & 1 & 11 & 0 & 91.7 \\
Bohai Rim region of China & 0 & 0 & 6 & 100.0 \\
Percentage (\%) & 45.5 & 36.4 & 18.2 & 93.9 \\
\hline
\end{tabular}

$$
\begin{aligned}
\operatorname{logit} p_{1}= & 17.130+1.506 x_{1}-0.372 x_{2}-5.343 x_{3}-4.632 x_{4}-1.883 x_{5}-2.199 x_{6} \\
& -4.932 x_{7}-5.439 x_{8}-4.909 x_{9}-4.817 x_{10}-4.750 x_{11}-4.626 x_{12}, \\
\operatorname{logit} p_{2}= & 22.543+4.042 x_{1}+3.115 x_{2}-2.835 x_{3}-2.711 x_{4}-0.876 x_{5}-2.086 x_{6} \\
& -3.294 x_{7}-3.421-3.343 x_{9}-2.640 x_{10}-2.708 x_{11}-1.491 x_{12}, \\
\operatorname{logit} p_{3}= & \text { (reference group). }
\end{aligned}
$$

Additionally, to validate whether the model shows adequate practical meaning, it is necessary to test the goodness of fit, pseudo $R$-squared, and likelihood ratio of the model. The tests (including the Pearson chi-square test and the deviance chi-square test) of goodness of fit can test whether the model fits the original data, or not. If the significance level exceeds 0.05 , the fitting effect is favourable. The pseudo $R$-squared value can verify the degree of explanation offered by the model for information contained in its original variables, which is shown in Cox, Nagelkerke and McFadden pseudo $R$-squared values. The closer the result is to 1 , the better the explanation. The likelihood ratio test measures the contribution of original variables to the model. If the significance level is lower than 0.05 , the contribution of original variables is high.

According to the test result (Table 8) obtained through use of the logistic regression model, the goodness of fit, pseudo $R$-squared, and likelihood ratio of the model all satisfy test requirements. This indicates that the extracted principal components PCA1, PCA2, and PCA3 also retain key information about the data while effectively realising dimension reduction, which makes a significant contribution to the construction of the logistic regression model. The final result obtained through model regression is also meaningful.

3.4.3. Validation of Discriminatory Effect of the Model. By taking IR CAPs of 33 original asphalt samples as verification samples, the discrimination effect obtained through the multinomial logistic regression model in multivariate statistical analysis was evaluated by applying formula (13). The discrimination result of multinomial logistic regression in multivariate statistical analysis is shown in Table 9.

As shown in Table 9, discrimination accuracies of 15, 12, and six asphalt samples separately produced by crude oil sourced from the Middle East, South America, and Bohai Rim region of China are $93.3 \%, 91.7 \%$, and $100 \%$, respectively. The comprehensive discrimination accuracy is $93.9 \%$. The above result showed that multivariate logistic regression analysis based on PCA can rapidly discriminate the origins of asphalt.

\section{Conclusions}

Based on ATR-FTIR technology, the infrared spectra of 33 kinds of asphalt produced by crude oil from the Middle East, South America, and Bohai Rim region of China were collected. Furthermore, the 12 selected CAPs of infrared spectra were analysed by multivariate statistics. The comprehensive accuracy of the logistic regression model based on PCA in discriminating asphalt, which were produced by crude oil from three different regions reached 93.9\%. The results indicated that the combination of ATR-IR spectral analysis and multivariate statistics can accurately and nondestructively discriminate between different crude oil source of asphalt. Moreover, the method shows some remarkable advantages, including ease of operation, rapidity, and high 
accuracy, which is important when controlling the origins and quality of asphalt and improving the performance thereof.

The method provided in this paper is suitable for the oil source identification of base asphalt produced by crude oil from different regions and can also provide reference for other kinds of asphalt, such as polymer-modified asphalt. However, the accuracy and applicability of this method need to be further improved. In particular, whether the asphalt produced by crude oil mixing from different regions can be effectively identified needs further research.

\section{Data Availability}

The data used to support the findings of this study are available from the corresponding author upon request.

\section{Conflicts of Interest}

The authors declare that they have no conflicts of interest.

\section{Acknowledgments}

This work was financially supported by the National Natural Science Foundation of China (no. 51608511), Key R\&D Project of Shandong Province (2018GGX105013), and Project of Science and Technology Support for Youth Entrepreneurship in Colleges and Universities of Shandong Province (2019KJG004). The authors would like to acknowledge many coworkers, students, and laboratory assistants for providing technical help on instrument analysis.

\section{References}

[1] S. Zhao and J. Liu, "Using recycled asphalt pavement in construction of transportation infrastructure: Alaska experience," Journal of Cleaner Production, vol. 177, pp. 155-168, 2018.

[2] Y. Tan and M. Guo, "Using surface free energy method to study the cohesion and adhesion of asphalt mastic," Construction and Building Materials, vol. 47, pp. 254-260, 2013.

[3] P. Li, J. Liu, and S. Zhao, "Implementation of stress-dependent resilient modulus of asphalt-treated base for flexible pavement design," International Journal of Pavement Engineering, vol. 19, no. 5, pp. 439-446, 2018.

[4] F. C. G. Martinho and J. P. S. Farinha, "An overview of the use of nanoclay modified bitumen in asphalt mixtures for enhanced flexible pavement performances," Road Materials and Pavement Design, vol. 20, no. 3, pp. 671-701, 2019.

[5] F. Xiao, D. Ma, J. Wang, D. Cai, L. Lou, and J. Yuan, "Impacts of high modulus agent and anti-rutting agent on performances of airfield asphalt pavement," Construction and Building Materials, vol. 204, pp. 1-9, 2019.

[6] Y. Sun, W. Wang, and J. Chen, "Investigating impacts of warm-mix asphalt technologies and high reclaimed asphalt pavement binder content on rutting and fatigue performance of asphalt binder through MSCR and LAS tests," Journal of Cleaner Production, vol. 219, pp. 879-893, 2019.

[7] M. Guo, Y. Tan, D. Luo et al., "Effect of recycling agents on rheological and micromechanical properties of SBS-modified asphalt binders," Advances in Materials Science and Engineering, vol. 2018, Article ID 5482368, 12 pages, 2018.
[8] W. Y. Fan, X. Xin, M. Liang et al., "Study on rheology and storage stability of modified asphalt," Journal of China University of Petroleum, vol. 39, pp. 165-170, 2015.

[9] M. Lagos-Varas, D. Movilla-Quesada, J. P. Arenas et al., "Study of the mechanical behavior of asphalt mixtures using fractional rheology to model their viscoelasticity," Construction and Building Materials, vol. 200, pp. 124-134, 2019.

[10] P. Wang, Z. J. Dong, Y. Q. Tan et al., "Effect of multi-walled carbon nanotubes on the performance of styrene-butadienestyrene copolymer modified asphalt," Materials and Structures, vol. 50, no. 1, p. 17, 2017.

[11] M. Guo, Y. Tan, L. Wang, and Y. Hou, "Diffusion of asphaltene, resin, aromatic and saturate components of asphalt on mineral aggregates surface: molecular dynamics simulation," Road Materials and Pavement Design, vol. 18, no. 3, pp. 149-158, 2017.

[12] Y. Xue, Z. Ge, F. Li, S. Su, and B. Li, "Modified asphalt properties by blending petroleum asphalt and coal tar pitch," Fuel, vol. 207, pp. 64-70, 2017.

[13] V. Y. Pivsaev, P. E. Krasnikov, A. A. Pimenov, and D. E. Bykov, "Enhancement of adhesive properties of road asphalts, waste oil processing products," Petroleum Chemistry, vol. 55, no. 1, pp. 80-83, 2015.

[14] M. N. Siddiqui, "NMR fingerprinting of chemical changes in asphalt fractions on oxidation," Petroleum Science and Technology, vol. 28, no. 4, pp. 401-411, 2010.

[15] M. N. Siddiqui, "NMR finger printing of chemical changes in asphalt fractions on oxidation," Petroleum Science and Technology, vol. 27, no. 17, pp. 2033-2045, 2009.

[16] R. Ren, K. Han, P. Zhao et al., "Identification of asphalt fingerprints based on ATR-FTIR spectroscopy and principal component-linear discriminant analysis," Construction and Building Materials, vol. 198, pp. 662-668, 2019.

[17] C. M. Xu, Y. Liu, S. Q. Zhao et al., "Analysis of heteroatomic compounds in petroleum asphaltenes by high resolution mass spectrometry," Journal of China University of Petroleum, vol. 37, pp. 190-195, 2013.

[18] Q. F. Wu and Z. C. Tang, "Study on the determinants of economic compensation for ecological damage caused by oil spill at sea," Journal of China University of Petroleum, vol. 34, pp. 1-8, 2018.

[19] Y. Zhong, H. Wang, G. Lu, Z. Zhang, Q. Jiao, and Y. Liu, "Detecting functional connectivity in fMRI using PCA and regression analysis," Brain Topography, vol. 22, no. 2, pp. 134-144, 2009.

[20] M. Kharbach, R. Kamal, M. A. Mansouri et al., "Selected-ion flow-tube mass-spectrometry (SIFT-MS) fingerprinting versus chemical profiling for geographic traceability of Moroccan argan oils," Food Chemistry, vol. 263, pp. 8-17, 2018.

[21] P. Y. Zhou, C. S. Chen, J. J. Ye et al., "Combining molecular fingerprints with multidimensional scaling analyses to identify the source of spilled oil from highly similar suspected oils," Marine Pollution Bulletin, vol. 93, no. 1-2, pp. 121-129, 2015.

[22] A. Ismail, M. E. Toriman, H. Juahir et al., "Chemometric techniques in oil classification from oil spill fingerprinting," Marine Pollution Bulletin, vol. 111, no. 1-2, pp. 339-346, 2016.

[23] J. M. Bayona, C. Domínguez, and J. Albaigés, "Analytical developments for oil spill fingerprinting," Trends in Environmental Analytical Chemistry, vol. 5, pp. 26-34, 2015.

[24] E. C. Y. Chan, P. K. Koh, M. Mal et al., "Metabolic profiling of human colorectal cancer using high-resolution magic angle spinning nuclear magnetic resonance (HR-MAS NMR) spectroscopy and gas chromatography mass spectrometry 
(GC/MS)," Journal of Proteome Research, vol. 8, no. 1, pp. 352-361, 2009.

[25] Y. Li, S. Wu, and S. Amirkhanian, "Investigation of the graphene oxide and asphalt interaction and its effect on asphalt pavement performance," Construction and Building Materials, vol. 165, pp. 572-584, 2018.

[26] G. Xu and H. Wang, "Molecular dynamics study of oxidative aging effect on asphalt binder properties," Fuel, vol. 188, pp. 1-10, 2017.

[27] N. Nciri and N. Cho, "A thorough study on the molecular weight distribution in natural asphalts by gel permeation chromatography (GPC): the case of Trinidad lake asphalt and asphalt ridge bitumen," Materials Today: Proceedings, vol. 5, no. 11, pp. 23656-23663, 2018.

[28] D. O. Larsen, J. L. Alessandrini, A. Bosch, and M. S. Cortizo, "Micro-structural and rheological characteristics of SBS-asphalt blends during their manufacturing," Construction and Building Materials, vol. 23, no. 8, pp. 2769-2774, 2009.

[29] H. Yao, Q. Dai, and Z. You, "Fourier transform infrared spectroscopy characterization of aging-related properties of original and nano-modified asphalt binders," Construction and Building Materials, vol. 101, pp. 1078-1087, 2015.

[30] F. Zhang, J. Yu, and J. Han, "Effects of thermal oxidative ageing on dynamic viscosity, TG/DTG, DTA and FTIR of SBS-and SBS/sulfur-modified asphalts," Construction and Building Materials, vol. 25, no. 1, pp. 129-137, 2011.

[31] A. Dazzi, C. B. Prater, Q. Hu, D. B. Chase, J. F. Rabolt, and C. Marcott, "AFM-IR: combining atomic force microscopy and infrared spectroscopy for nanoscale chemical characterization," Applied Spectroscopy, vol. 66, no. 12, pp. 1365-1384, 2012.

[32] M. Lopes, V. Mouillet, L. Bernucci, and T. Gabet, "The potential of attenuated total reflection imaging in the mid-infrared for the study of recycled asphalt mixtures," Construction and Building Materials, vol. 124, pp. 1120-1131, 2016.

[33] Y. Xiong, G. Chen, S. Guo, and G. Li, "Lifetime prediction of NBR composite sheet in aviation kerosene by using nonlinear curve fitting of ATR-FTIR spectra," Journal of Industrial and Engineering Chemistry, vol. 19, no. 5, pp. 1611-1616, 2013.

[34] G. Y. Chen and S. E. Qian, "Denoising of hyperspectral imagery using principal component analysis and wavelet shrinkage," IEEE Transactions on Geoscience and Remote Sensing, vol. 49, no. 3, pp. 973-980, 2010.

[35] S. Tonidandel and J. M. LeBreton, "Determining the relative importance of predictors in logistic regression: an extension of relative weight Analysis," Organizational Research Methods, vol. 13, no. 4, pp. 767-781, 2010.

[36] R. Azen and N. Traxel, "Using dominance analysis to determine predictor importance in logistic regression," Journal of Educational and Behavioral Statistics, vol. 34, no. 3, pp. 319-347, 2009.

[37] J. Adler and I. Parmryd, "Quantifying colocalization by correlation: the Pearson correlation coefficient is superior to the Mander's overlap coefficient," Cytometry Part A, vol. 77A, no. 8, pp. 733-742, 2010.

[38] A. Soberón and W. Stute, "Assessing skewness, kurtosis and normality in linear mixed models," Journal of Multivariate Analysis, vol. 161, pp. 123-140, 2017.

[39] A. D. Ho and C. C. Yu, "Descriptive statistics for modern test score distributions," Educational and Psychological Measurement, vol. 75, no. 3, pp. 365-388, 2015. 